\title{
Dissociative disorder in Mayer Rokitansky Küster Hauser syndrome with pulmonary agenesis: a case report
}

\author{
Sulochana Joshi ${ }^{{ }^{*}} \mathbb{D}$, Anup Raj Bhandari', Prashant Shrestha² and Rabi Shakya ${ }^{1}$
}

\begin{abstract}
Background: The Mayer Rokitansky Küster Hauser (MRKH) syndrome is a rare congenital disorder characterized by the absence of uterus and vagina in a patient who is phenotypically a female, with 46 XX karyotypes. It affects 1 in 4000 to 5000 female new-borns. Pulmonary agenesis is a rare association in this MRKH syndrome. Females with MRKH face various mental health issues and psychological disturbances, including dissociative disorder which is a stressrelated psychiatric disorder. Dissociative disorder in MRKH syndrome is under-recognized and under-treated.

Case presentation: A 23-year-old unmarried woman presented to the emergency forabnormal behaviour attack. Recurrent episodes of dissociative convulsions were present most of the time with MRKH syndrome as the pertinent stressor. Both antidepressants and psychotherapy helped to decrease the frequency of dissociative convulsions and come interms with the syndrome.

Conclusion: This case describes dissociative disorder as the presentation and comorbid condition of MRKH syndrome and the impact of MRKH syndrome on the patient. We attempt to explain the occurrence of dissociative disorder in MRKH syndrome and the results of under-recognition and under-treatment of the same. We aim to highlight the presence of commonly treatable conditions associated with a rare syndrome and its effect when untreated and unrecognized.
\end{abstract}

Keywords: Dissociative disorder, Mayer Rokitansky Küster Hauser syndrome, Psychological disturbance, Treatment, Case report

\section{Background}

The Mayer Rokitansky Küster Hauser (MRKH) syndrome is a rare congenital disorder characterized by the absence of uterus and vagina in a patient who is phenotypically a female, with $46 \mathrm{XX}$ karyotypes. It has an autosomaldominant inheritance pattern and is a result of the developmental malformation of Mullerian ducts [1]. It affects 1 in 4000 to 5000 female new-borns [1, 2]. Pulmonary agenesis is a rare association in the MRKH syndrome [2-4]. Psychiatric comorbidities among these patients are

\footnotetext{
${ }^{*}$ Correspondence: sulochanajoshi01@gmail.com

${ }^{1}$ Department of Psychiatry, Patan Academy of Health Sciences, Lalitpur, Nepal

Full list of author information is available at the end of the article
}

less frequently reported and psychiatric care is neglected. However, females with this syndrome face various mental health issues including dissociative disorder $[1,5]$. Dissociative disorder is a stress-related psychiatric disorder with disruption of normal integration between memories of the past, awareness of identity, and immediate sensations and control of bodily movements leading to various kinds of manifestations that resemble physical and neurological conditions [6]. In this case report, we discuss one of the presentations of MRKH syndrome, its underrecognition, and under-treatment. This case highlights the need to address and manage the psychiatric problems associated with the congenital anomalies of the reproductive system. 


\section{Case presentation}

A 23-year-old female, a receptionist by occupation, presented at the emergency of our hospital with complaints of recurrent abnormal behaviour attacks for three and half years and current recurrence for last 10 days. These behaviour attacks lasted minutes to hours. During such behaviour attacks, she responded to verbal commands and manifested abnormal behaviors, such as scratching, pulling her hair, banging her head, and hitting people nearby. She talked mimickingthe voice of her deceased father and claimed to be his soul. She tightened her limbs and moved them. These behaviour attacks were also accompanied by difficulty in breathing and apprehension.

On further inquiry, it was found that she had decreased sleep, restlessness, startled response, and worry. Her father had died of a brain tumor 2 years ago. She is the second child among four siblings and was living with her mother, 2 sisters, and a brother. She did not share a good relationship with her immediate elder sister. She expressed anger outbursts towards her elder sister for being in a better position than herself in all aspects. She had an uneventful birth history but was frequently ill during her childhood, mostly with chest infections. She was diagnosed with MRKH syndrome at 13 years of age. She then learned that she has only one lung on the right side, and she did not have a uterus, nor a vagina, and, therefore, cannot bear children. Her partner ended their 5-year relationship giving MRKH syndrome as the excuse leaving her emotionally traumatized. However, there was no history suggestive of persistent low mood or any abnormal thought or perceptual disturbance. During the mental state examination, she was uncooperative and irritable. She was diagnosed with Dissociative disorder (convulsion type) with adjustment disorder with MRKH syndrome.

She was started on Escitalopram $10 \mathrm{mg}$. The psychologist explored the triggers for her negative thoughts and planned for emotional handling of issues, anger management, and family-focused intervention. Her restlessness and worries improved but the improvement did not last long. She kept visiting emergency department in every 2-3 days, each time with increasing frequency of dissociative convulsions. The patient had 8-10 episodes of dissociative convulsions in a day. After multiple visits to emergency department, she was admitted to the Psychiatry department for further management. In the ward, the patient was consulted with the gynecology team to review the diagnosis of MRKH syndrome and possible treatment options of the same. The gynaecology team confirmed her diagnosis as MRKH syndrome with history of amenorrhea and Ultrasonography findings of abdomen and pelvis and planned an intravenous urogram, which she refused along with other related interventions. She and her mother did not want to spend any more money on the investigations related to MRKH syndrome. Baseline investigations (Complete Blood Count, Renal Function Test, Urine Routine, and Microscopic Examination and Thyroid Function Test) were normal. The ultrasound scan did not show the right kidney, and her uterus was hypotrophic (of size $2.2 \mathrm{~cm} \mathrm{X} 0.8 \mathrm{~cm} \mathrm{X} 1 \mathrm{~cm}$ ). Chest X-ray showed single right lung. During the hospital stay, the psychologist had multiple sessions with her. Initial sessions were focused on assessing the knowledge of the caretaker and patient regarding her illness. Different ongoing stressors were explored and psycho-education was done about the nature of illness and the possible role of the family in the management of her symptoms. Her range of coping behaviors was explored using the Brief Cope Scale which revealed low assertiveness and avoidance-focused coping. Her mother was her primary caretaker and was also involved in the session to promote supportive interactions with her when she had dissociative convulsions. She was also trained on assertive communication and anger management skills to break the vicious cycle of interpersonal relationship issues with her family members and recurrence of dissociative convulsions and anger outbursts. There were marked improvements in the frequency of dissociative convulsions and anger outbursts with multiple sessions by the psychologist and the cut down of secondary gains. The secondary gains were the love and attention from her mother which she was not given to that extent earlier. Her mother did not complain about her anger outbursts and temper tantrums after episodes of dissociative convulsion. She was also introduced to cognitive behavior therapy (CBT) and planned for further sessions. She was discharged after 1 week with Escitalopram $10 \mathrm{mg}$ and Clonazepam $0.25 \mathrm{mg}$ on SOS basis.

However, the patient got readmitted for the second time after 1 week of improvement. She had developed sleep disturbance and decreased appetite. She had left her job but nobody asked her to resume her job. Mental Status Examination revealed irritability, restlessness, and preoccupation with illness. The patient used to think about her congenital condition and perceived this condition as a reason for any of her activity, events, failures, inability, difference from others. This congenital condition made her feel inferior and angry. Further assertiveness skill was explored and explained by the psychologist. The frequency of dissociative convulsion decreased in frequency and duration during the hospital stay. She was discharged with Sodium Valproate $300 \mathrm{mg}$ which was optimized to $500 \mathrm{mg}$ and Escitalopram $10 \mathrm{mg}$ after 1 week of her hospital stay.

She was doing well for 1 year when she again got admitted for the third time for 1 week for similar problems 
along with the attempts of self-harm (wrist cutting). The attempts of self-harm by wrist cutting were done because of restlessness and anger outbursts when things did not happen according to her. During this admission, she had developed a hearing deficit in her left ear. She had expressed that she had difficulty hearing from her left ear which was observable and evident during the interaction as one had to repeat and speak in loud volume to communicate. However, she refused the ENT consultation despite explaining the need for evaluation by an ENT specialist for the diagnosis and management. Her symptoms gradually improved but continued having recurrent episodes of dissociative convulsions in the interval of weeks to months. The psychologist continued focused sessions on distress tolerance, the Antecedents, Behavior, Consequences (ABC) Model explored and explained along with supportive psychotherapy. Despite all the efforts, regular follow-up in months, and compliance with the medications, whenever any issues occurred about her physical condition, she had episodes of dissociative convulsions.. Nevertheless, these decreased with time to minimal episodes of 1-2 in a week.

\section{Discussion}

Our case report highlights the occurrence of dissociative disorder in MRKH syndrome. The disorder has not been reported in MRKH syndrome so far. Here the patient suffers from repeated dissociative convulsions. Despite knowing about the congenital abnormality at an early age, the patient and the family did not have adequate information about the mental health issues regarding the MRKH syndrome. Our patient had multiple chest infections frequently starting from early childhood, as well as relationship issues and questions regarding her fertility and life with a partner during her late teens. In addition, for the last three and half years the patient had multiple (8-10 episodes in a day) and frequent episodes of dissociative convulsions which decreased to a few (1-2 episodes in a week) episodes with treatment by CBT, supportive psychotherapy and antidepressants for the coexisting adjustment disorder. Nevertheless, these dissociative convulsions are still occurring infrequently and issues with family members, workplace all are impacting her life. The studies have shown that the psychological impact of the syndrome increases with time and so does the suffering. [7, 8] This was no different in our case as the patient suffered due to the ongoing multiple dissociative convulsions, under-recognition of these by her family, her physical condition and another rare association of the presence of single lung (lung agenesis) to her rare congenital condition. The patient had refused further gynaecology consultations and was not interested in any treatment regarding the same. This created an unapproachable situation in the beginning.

However, dissociative disorders are common and convulsion subtypes are frequent manifestations. Though the literature on the mental health issues and psychological impact of the syndrome is scarce, reports of psychiatric illness, such as depression, suicide, schizophrenia, psychosis, psychological disturbances have been reported in MRKH syndrome [7, 9-12].] Dissociative convulsions have not been reported so far in the available literature. Dissociative disorder per se is not an unexpected psychiatric disorder to occur in a patient with MRKH syndrome. This physical abnormality acts as the obvious and pertinent stressor for the dissociation phenomenon. MRKH syndrome has enough impetus for the development of dissociation such as the age at which one is diagnosed and the effect of diagnosis, the psychosocial development, development of personality, infertility and sexual activity difficulties, emotional disturbances [7, 12-14] To our knowledge, our case is the first one to report on the dissociative disorder in the MRKH syndrome. However, this commonly occurring dissociative disorder is neglected and not considered important such as other kinds of psychiatric illnesses associated with MRKH syndrome [7, 9, 12]. This could be because of under-recognition of the dissociative disorder. The other reason could be the more emphasis on the rarity of the syndrome which overshadows the treatable common comorbidity.

MRKH syndrome with a dissociative disorder is challenging to manage as much cannot be done to the stressor, the congenital abnormality. The holistic approach is the key to its management with an emphasis on the continuous treatment of the psychological consequences of the syndrome [14-16]. Though the dissociative disorder is well evidenced psychiatric disorder, patients with these disorders continue to be under- or misdiagnosed, under- or mistreated, and insufficiently respected [17]. The comorbidities usually bring these patients for the treatment rather than the disorder itself. In our patient, the frequent visit to emergency and repeated admission helped in providing better psychoeducation about the disorder to the family and conduct supportive psychotherapy and work in liaison with the gynaecologist for the possible treatment options emphasizing the need for teamwork and holistic treatment. This case illustrates the common condition in the rare syndrome and how it is equally important and challenging to treat the common condition for the overall management of the patient with the rare syndrome. 


\section{Conclusion}

This case emphasizes the need to address and manage the psychological problems associated with the congenital anomalies of the reproductive system. It is imperative to assess these women regularly by mental health experts at diagnosis and at intervals to avoid missing these common treatable comorbidities. Dissociative disorders are neglected disorders as well as are not covered in the psychiatric assessment tools. Routine assessment of these disorders in psychiatric evaluation using structured tools would result in early recognition, awareness about childhood trauma, prevention of adverse childhood experiences and lifelong clinical consequences.

\section{Abbreviations}

MRKH: Mayer Rokitansky Küster Hauser; CBT: Cognitive Behaviour Therapy; ABC Model: Antecedents Behaviour Consequences Model.

\section{Acknowledgements}

Not applicable.

\section{Authors' contributions}

SJ conceived the study and participated in itsdesign. SJ participated in coordination and drafting the manuscript. PS and ABcontributed to drafting the manuscript. RS contributed in revising themanuscript critically. All authors read and approved the final manuscript.

\section{Funding}

Not applicable.

\section{Availability of data and materials}

Not applicable.

\section{Declarations}

Ethics approval and consent to participate

Not applicable.

\section{Consent for publication}

Written informed consent was obtained from the patient and her mother for the publication of this case report.

\section{Competing interests}

The authors declare that they have no competing interests.

\section{Author details}

'Department of Psychiatry, Patan Academy of Health Sciences, Lalitpur, Nepal. ${ }^{2}$ Department of Gynaecology and Obstetrics, Patan Academy of Health Sciences, Lalitpur, Nepal.

Received: 22 July 2021 Accepted: 21 December 2021

Published online: 06 January 2022

\section{References}

1. Londra L, Chuong FS, Kolp L. Mayer-Rokitansky-Kuster-Hauser syndrome: a review. Int J Womens Health. 2015;7:865-70.

2. Shivalingappa SS, Shetty SB. Mayer-Rokitansky-Küster-Hauser (MRKH) syndrome with unilateral pulmonary agenesis - a rarity indeed: radiologic review. BJR Case Rep. 2016;2:2.

3. Bachh AA, Pulluri S, Beigh A, Raju C, Deshpande R. Pulmonary aplasia with unusual associations in a woman Iran. J Med Sci. 2014;39(2):148-51.
4. Acién P, Galán F, Manchón I, Ruiz E, Acién M, Alcaraz LA. Hereditary renal adysplasia, pulmonary hypoplasia and Mayer-Rokitansky-Küster-Hauser (MRKH) syndrome: a case report. Orphanet J Rare Dis. 2010;5(1):6.

5. Nunes RD. Mayer-Rokitansky-Kuster-Hauser syndrome-case report. Obstet Gynaecol Cases - Rev. 2015;2:3.

6. ICD-10 Version:2019.WHO. https://icd.who.int/browse10/2019/en\#/F40F48. Accessed 24 Jul 2020.

7. Heller-Boersma JG, Schmidt UH, Keith ED. Psychological Distress in Women With Uterovaginal Agenesis (Mayer-Rokitansky-Küster-Hauser Syndrome, MRKH). Psychosomatics. 2009;50(3):277-81.

8. Bargiel-Matusiewicz K, Kroemeke A. Personality traits and coping styles in women with Mayer-Rokitansky-Küster-Hauser syndrome. Arch Med Sci AMS. 2015;11(6):1244-9.

9. Gupta M, Kharb V. MRKH syndrome: psychological disturbances and suicide. J Indian Acad Forensic Med. 2012;34(1):3.

10. Bhad R, Chadda R, Kumar N, Goyal P. A rare association of schizophrenia and Mayer-Rokitansky-Küster-Hauser syndrome. Indian J Psychiatry. 2015;57(3):324-5

11. Chen N, Song S, Duan Y, Kang J, Deng S, Pan H, et al. Study on depressive symptoms in patients with Mayer-Rokitansky-Küster-Hauser syndrome: an analysis of 141 cases. Orphanet J Rare Dis. 2020;15(1):121.

12. Nath K, Boro B, Naskar S. A Rare Case of Mayer-Rokitansky-Kuster-Hauser syndrome presenting with acute psychosis. J Clin Diagn Res JCDR. 2016;10(4):3-4.

13. Heller-Boersma JG, Edmonds DK, Schmidt UH. A cognitive behavioural model and therapy for utero-vaginal agenesis (Mayer-Rokitansky-KüsterHauser syndrome: MRKH). Behav Cogn Psychother. 2009;37(4):449-67.

14. MI P, Tn E. Psychologic aspects of vaginal agenesis. J Reprod Med. 1985;30(4):340-4.

15. Committee on Adolescent Health Care. ACOG Committee Opinion No. 728: Müllerian Agenesis: Diagnosis, Management, And Treatment. Obstet Gynecol. 2018;131(1):e35-42.

16. Liszewska-Kapłon M, Strózik M, Kotarski Ł, Bagłaj M, Hirnle L. Mayer-Rokitansky-Küster-Hauser syndrome as an interdisciplinary problem. Adv Clin Exp Med. 2020;29(4):505-11.

17 Spiegel D. Recognizing traumatic dissociation. Am J Psychiatry. 2006;163(4):566-8.

\section{Publisher's Note}

Springer Nature remains neutral with regard to jurisdictional claims in published maps and institutional affiliations.

\section{Submit your manuscript to a SpringerOpen ${ }^{\odot}$ journal and benefit from:}

- Convenient online submission

- Rigorous peer review

- Open access: articles freely available online

- High visibility within the field

- Retaining the copyright to your article

Submit your next manuscript at $\boldsymbol{\nabla}$ springeropen.com 